\title{
Effect of aerobic exercise on blood pressure in men with hypertension: A randomized controlled study
}

\author{
Mitiku Daimo $^{1}, \odot$ Soumitra Mandal ${ }^{2}, \odot$ Mahmud Abdulkader $^{3}$, \\ Dhamodharan Mathivanan ${ }^{2}$
}

${ }^{1}$ Department of Sports Science, Hawassa College of Teachers Education, Hawassa-Ethiopia.

${ }^{2}$ Department of Sport Science, College of Natural and Computational Sciences, Mekelle University, Mekelle, Ethiopia.

${ }^{2}$ Institute of Medical Microbiology E Immunology, College of Medical Science, Mekelle University, Mekelle, Ethiopia.

\begin{abstract}
This study was aimed at determining the effects of aerobic exercise training on blood pressure in men hypertensive patients. To this end, the study employed a randomized controlled, parallel-group design.
\end{abstract} Accordingly, twenty-four male hypertensive patients with a mean age of $38 \pm 4$ years were assigned into two groups $(\mathrm{n}=12$ each): the exercise group means body mass index $26.8 \pm 1.54 \mathrm{~kg} / \mathrm{m} 2$ and the control group's mean body mass index $27.2 \pm 1.98 \mathrm{~kg} / \mathrm{m} 2$. The research participants' systolic blood pressure (SBP) and diastolic blood pressure (DBP) data were collected at baseline and after 16 weeks of the study period. The exercise group (EG) participants performed moderate-intensity aerobic exercise for 16 weeks three days per week. On the other hand, the control group (CG) participants did not take part in exercise intervention but continued their usual daily activities during the study period. Statistical analysis data collected was done using SPSS version 20. Analyses of withingroup and between-group comparisons were done using paired sample t-test and independent sample t-test, respectively, with the level of significance set at $\mathrm{P}<0.05$. The results of this analysis show that the EG has demonstrated significant within-group mean changes in SBP (-7.1 mm Hg; P<0.001), and in DBP $(-5.6 \mathrm{~mm} \mathrm{Hg}$; $\mathrm{P}<0.001)$ compared to baseline. The result in the CG was found insignificant within-group mean changes in SBP ($0.3 \mathrm{~mm} \mathrm{Hg}$; $\mathrm{P}=0.51)$, and in DBP $(-0.3 \mathrm{~mm} \mathrm{Hg} ; \mathrm{P}=0.39)$ compared to baseline. Moreover, between-group comparisons showed that mean SBP and DBP significantly reduced in EG compared to CG. Therefore, it can be concluded that 16 weeks, moderate-intensity and supervised aerobic exercise training significantly reduce blood pressure levels in stage 1 hypertensive patients.
Thus hypertensive patients could be encouraged to take part in moderate-intensity aerobic exercise training regularly.

Keywords. Aerobic exercise, blood pressure, hypertensive patients

\section{Introduction}

Hypertension is worldwide widespread and human beings' health problems (Cardoso et al., 2010; Kearney et al., 2005). In peoples sustained high blood pressure (HBP) affects primarily the aorta, heart, brain, kidneys, retina, and lower extremities (Parkinson, 2012). The lifestyle changes of modern people are related to growing urbanization and rising utilization of motorized transportation and these lifestyle alterations lead people to increase sedentary mode of life (Andreeva, 2014; Boreham \& Riddoch, 2001). Now a day there is a notable increase in urbanization in Ethiopia (Hailemariam et al., 2018).

An inactive lifestyle increases the risk of hypokinetic diseases, including hypertension (Ferrari, 2008). Inactivity is greatly connected with hypertension (Carnethon et al., 2003). Nshisso et al. (2012) reported that inactive lifestyles, expanding in low-income countries, including Ethiopia, causing speeding up in the occurrence of hypertension. From 2000 to 2010 the prevalence of hypertension increased by $16 \%$ in both men and women in Sub 
Saharan Africa and the prevalence of hypertension was (36.3\%) for women and (36.9\%) for men (Mills et al., 2016). The world health organization estimated that the prevalence of hypertension in Ethiopia is to be $35.2 \%$ in 2025 (WHO, 2011). In Ethiopia at present in the everyday life of the urban people, high attention is not given for physical exercise (Belay et al., 2013).

To encounter the increased prevalence of hypertension increased involvement in exercise intervention could be the main alternative in lowand middle-income countries (Whelton et al., 2002a). To reduce hypertension, regular participation in exercise training is an important nonpharmacological strategy (Pescatello et al., 2015; Chobanian et al., 2003; Whelton et al., 2002b).

According to the recommendations of ACSM, WHO, an international society of hypertension, national heart foundation and a committee on prevention, detection, evaluation, and treatment of HBP, increased physical activity is considered to be the first-line treatment strategy for mild and moderate hypertensive patients (Baster \& Baster, 2005). Aerobic exercise training modality is very important for the treatment and prevention of HBP and its complications (Borjesson et al., 2016; Cornelissen \& Smart, 2013; Cardoso et al., 2010; Iellamo \& Volterani, 2010).

To the best of our knowledge studies were not carried out in Ethiopia concerning aerobic exercise training as an alternative intervention approach for hypertensive patients. Hence, this study aimed to determine the effect of aerobic exercise training on $\mathrm{BP}$ in men with hypertension.

\section{Methods}

\section{Subjects}

Twenty-four male stage 1 hypertensive patients, age ranges between 31 to 45 years, individuals who provided written informed consent, participants who live in the area of study during the research and participants who are on monotherapy were included in the study. Whereas participants with known renal and cardiac problems, diabetic patients, participants who use lipid-altering medications and participants who were involved in a structured exercise program within the three-month gap before the study period were excluded from the study.

\section{Ethical Considerations}

This study was approved by the Health Research Ethics Review Committee of Health Sciences College of Mekelle University with Ref.ERC07752016. Before continuing the study written informed consent was obtained from all study participants after reading and explaining information sheets in their local language (Amharic) regarding procedures, confidentiality, and risks of the study. The study was conducted according to the recommendations of the Declaration of Helsinki.

\section{Study Design}

This study used a randomized, controlled, parallelgroup design. Twenty-four hypertensive males, who fulfill inclusion, exclusion criteria were randomly assigned into an exercise group $(n=12)$ and a control group $(n=12)$ after assessment for eligibility. Flow chart of study participants presented in Figure 1.

\section{Data collection}

Baseline data on BP were collected after a minimum of 48 hours of vigorous physical activity (exercise training) and after 12 hours overnight fasting in the morning between $8 \mathrm{am}$ to $10 \mathrm{am}$. Resting SBP and DBP was taken by using an Omron digital sphygmomanometer. The participant seated on the chair, leg uncrossed and arm placed on the table at the height of the heart with the palm facing upward. The first measurement was taken after a person sits down for at least 10 minutes and the second and third measurements were taken after 3 minutes of rest between the readings. The precision of the measurement was kept by using three readings 
within a 3-minute interval and finally, BP values used for analysis were the mean of the three readings.

\section{Training protocol}

The participants in the exercise group (EG) performed aerobic exercise (brisk walking) for 16 consecutive weeks, three days per week. An exercise physiologist supervised aerobic exercise training. The participants in the control group (CG) continued their usual activities during the period of study and were accomplished medical symptom's questionnaire forms monthly for the study period, but did not take part in any structured exercise training. The aerobic exercise training protocol is presented in Table 1.

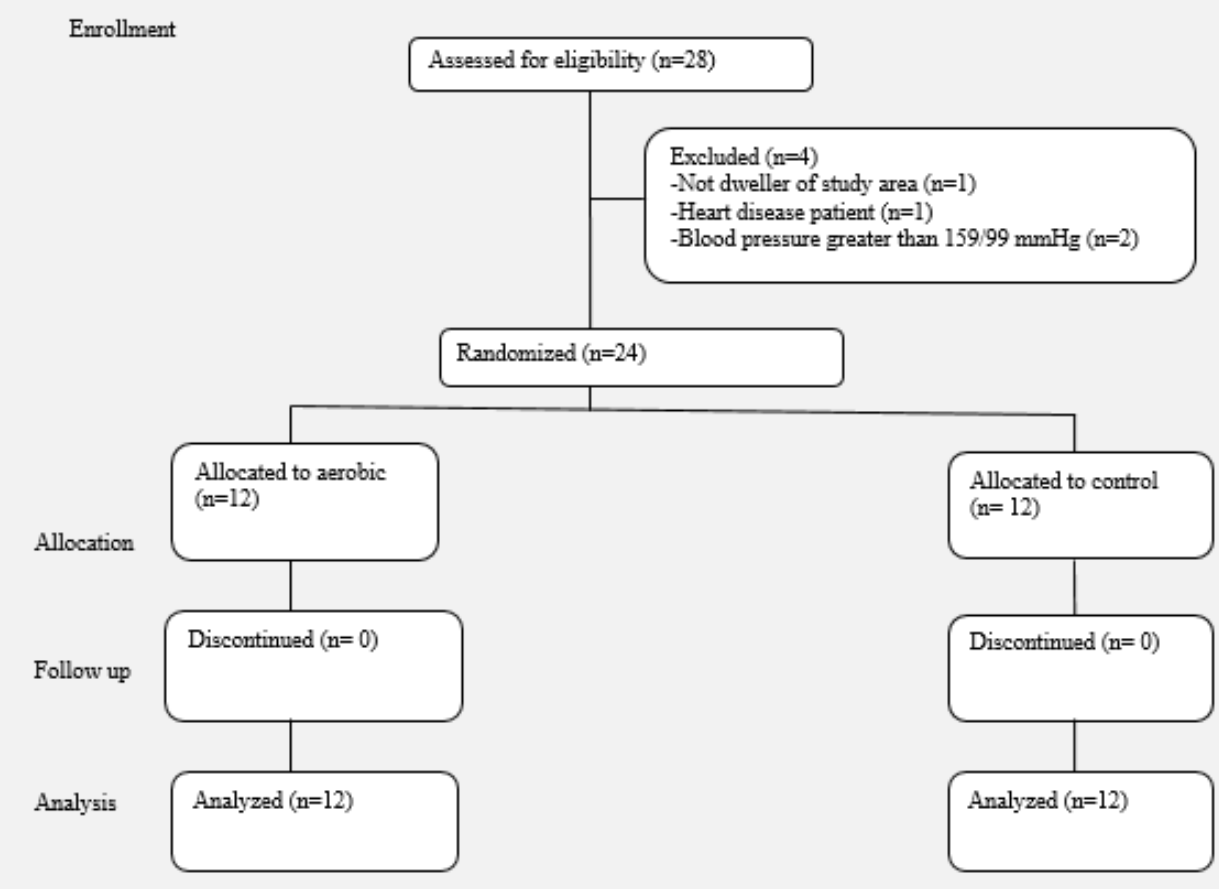

Figure 1. Flow chart of study participants.

\section{Table 1}

Aerobic exercise training protocol for hypertensive patients.

\begin{tabular}{lccc}
\hline Weeks & Duration of Exercise $(\mathrm{min})$ & Intensity & Frequency of Days per Week \\
\hline $1-2$ & 30 & $30 \%$ of HRR & 3 \\
$3-4$ & 30 & $35 \%$ of HRR & 3 \\
$5-6$ & 35 & $40 \%$ of HRR & 3 \\
$7-8$ & 35 & $45 \%$ of HRR & 3 \\
$9-10$ & 40 & $50 \%$ of HRR & 3 \\
$11-12$ & 40 & $55 \%$ of HRR & 3 \\
$13-14$ & 45 & $60 \%$ of HRR & 3 \\
$15-16$ & 45 & $60 \%$ of HRR &
\end{tabular}




\section{Statistical Analysis}

Data entry and analysis were done using the Statistical Package for Social Sciences (SPSS) Version 20. The mean values and standard deviations were tabulated for SBP and DBP. A comparison of quantitative variables at the pre-intervention and after post-intervention of the same group was analyzed with paired sample t-test. Comparison of continuous variables between the intervention and control group was done using an independent sample t-test. Finally, the statistical significance was set at $\mathrm{P}<0.05$.

\section{Results}

Twenty-four hypertensive males were studied. All study participants completed the study and analyzed it. For hypertensive patients treated with aerobic exercise, mean and SD of age (in years), height (in meters), weight (in kilograms),body mass index (in $\mathrm{kg} / \mathrm{m}^{2}$ ), $\mathrm{SBP}$ (in $\mathrm{mmHg}$ ) and DBP (in $\mathrm{mmHg}$ ) were 38.42 (4.69), 1.68 (0.07), 77(9.57), 26.8 (1.54),142.8 (2.92) and 91.2 (1.59) respectively, and for the control group the mean (SD) of age (in years), height (in meters), weight (in kilograms), body mass index (in $\left.\mathrm{kg} / \mathrm{m}^{2}\right), \mathrm{SBP}($ in $\mathrm{mmHg}$ ) and DBP (in $\mathrm{mmHg}$ ) were 37.6 (3.6), 1.69 (0.05), 77.7 (8.75), 27.2 (1.98), 142.5 (3.089) and 91.08 (1.16), respectively. There were no significant baseline differences between study groups in all measured variables $(P>0.05)$. The descriptive characteristics of the study groups are presented in Table 2.

\section{Table 2}

Descriptive characteristics of groups.

\begin{tabular}{lccccc}
\hline \multirow{2}{*}{ Variables } & \multicolumn{2}{c}{ Aerobic Group } & \multicolumn{2}{c}{ Control Group } & \multirow{2}{*}{$p$} \\
\cline { 2 - 4 } & Mean & SD & Mean & SD & \\
\hline Age (years) & 38.42 & 4.69 & 37.6 & 3.60 & 0.10 \\
Height (meters) & 1.68 & 0.07 & 1.69 & 0.05 & 0.92 \\
Weight (kg) & 77 & 9.57 & 77.7 & 8.75 & 0.65 \\
Body mass index $\left(\mathrm{kg} / \mathrm{m}^{2}\right)$ & 26.8 & 1.54 & 27.2 & 1.98 & 0.42 \\
SBP & 142.8 & 2.92 & 142.5 & 3.09 & 0.78 \\
DBP & 91.2 & 1.59 & 91.08 & 1.16 & 0.88 \\
\hline
\end{tabular}

$S D=$ Standard Deviation

Table 3

Paired sample t-test of the blood pressure within the aerobic and control groups.

\begin{tabular}{llcccccc}
\hline Groups & Variables & Baseline & 16 weeks & MD & $\mathrm{t}$ & $\mathrm{df}$ & $p$ \\
\hline Aerobic & SBP & $142.8 \pm 2.92$ & $135.7 \pm 3.28$ & -7.1 & -10.843 & 11 & $0.001^{*}$ \\
& DBP & $91.2 \pm 1.59$ & $85.6 \pm 2.94$ & -5.6 & -8.818 & 11 & $0.001^{*}$ \\
Control & SBP & $142.5 \pm 3.089$ & $142.2 \pm 3.16$ & -0.3 & -0.692 & 11 & 0.51 \\
& DBP & $91.08 \pm 1.16$ & $90.75 \pm 1.54$ & -0.3 & -0.886 & 11 & 0.39 \\
\hline
\end{tabular}

${ }^{*}$ The mean difference is significant at $p<0.05$. Values are presented as Mean $\pm S D . S B P=$ Systolic blood pressure;

$D B P=$ diastolic blood pressure; $M D=$ mean difference. 


\section{Table 4}

Mean and SD of blood pressure change in study participants between aerobic and control groups using independent sample t-test.

\begin{tabular}{llccrc}
\hline Variables & Groups & Mean \pm SD & t & df & \\
\hline SBP & Aerobic & $135.7 \pm 3.28$ & -4.942 & 22 & $0.001^{*}$ \\
& Control & $142.2 \pm 3.16$ & & 22 & $0.001^{*}$ \\
DBP & Aerobic & $85.6 \pm 2.94$ & -5.393 & & \\
& Control & $90.75 \pm 1.54$ & & \\
\hline
\end{tabular}

* The mean difference is significant at $p<0.05$.

$S B P$, Systolic blood pressure; DBP, diastolic blood pressure.

Mean SBP and DBP significantly reduced in EG (by $-7.1 \mathrm{mmHg}, \mathrm{P}<0.001$ and by $5.6 \mathrm{mmHg}, \mathrm{P}<0.001$ ), respectively compared to baseline. Mean SBP and DBP not significantly reduced in CG compared to baseline. The results of the paired sample t-test presented in Table 3. The EG had shown a significant mean reduction in SBP and DBP compared to CG after sixteen weeks of exercise intervention. The results of the independent sample t-test presented in Table 4.

\section{Discussion}

The finding of the current study indicated that resting SBP and DBP were significantly decreased in the EG when compared to baseline (Table 3) and the CG (Table 4). This is likely because, the decreased sympathetic nervous system activity and possible normalization of arteriole morphology may have a contribution to reducing peripheral resistance to blood flow and thereby lowering blood pressure (O'Sullivan \& Bell, 2000). Further aerobic exercise training reduces blood pressure by decreasing vascular resistance Cornelissen \& Smart (2013), Halliwill et al. (2013), Fagard (2006), Cornelissen \& Fagard (2005) by rising vasodilatation and lumen diameter (Hamer, 2006), by decreasing endothelial-1 that is a powerful vasoconstrictor, and by raising nitric oxide which is a strong vasodilator (Maeda et al., 2001).
The statistically significant reductions found in resting blood pressure among adult hypertensive patients in the current study have high clinical importance. Some studies reported that a decrease of $5 \mathrm{mmHg}$ in SBP reduces the risk of coronary heart disease by $9 \%$ and the risk of stroke by $14 \%$ (Chobanian et al., 2003; Whelton et al., 2002b). Our study demonstrated a mean SBP reduction of 7.1 $\mathrm{mmHg}$ due to aerobic exercise training according to the paired-sample t-test result (Table 3). This indicated that participants of the EG have reduced coronary artery disease risk by $12.8 \%$ and stroke risk by $19.9 \%$. So this study has vital clinical significance in addition to statistical significance for the participants of the study in improving their blood pressure.

In agreement with the present study finding studies conducted by Swati et al. (2015), Collier et al. (2008), Westhoff et al. (2008), Fagard \& Cornelissen (2007), Fagard (2006), Cornelissen \& Fagard (2005), Isdebska et al. (2004) reported that both resting SBP and DBP significantly reduced at the end of the aerobic exercise intervention compared to baseline. The findings of our study also supported by Gholamrezai et al. (2014), Lima et al. (2014), Cornelissen \& Smart (2013), Lamina (2010), Tsai et al. (2004), Tsai et al. (2002) those reported significant reduction in resting $\mathrm{SBP}$ and $\mathrm{DBP}$ in the EG compared to CG after the intervention.

However, some studies found no significant changes in SBP and DBP after 8 weeks to 16 weeks of 
aerobic exercise intervention (Lima et al., 2012; Bateman et al., 2011). The variations may be attributed to differences in ethnicity, age of the participants and baseline blood pressure level of the participants.

Application of randomized controlled studies, higher adherence level of the participants in the exercise, provision of an alternative approach to treat hypertension in Ethiopia for the first time are the strengths of the study. Whereas, using a small sample size is a limitation of this study. So, future studies should be conducted using large sample sizes.

\section{Conclusion}

The aerobic exercise group had shown significant decreases in SBP and DBP when compared with the baseline data as well as the control group after 16weeks of moderate-intensity aerobic exercise intervention in stage 1 hypertensive patients. Therefore, it can be concluded that 16 weeks, moderate-intensity and supervised aerobic exercise intervention significantly reduce BP levels in stage 1 hypertensive patients. Thus hypertensive patients could be encouraged to take part in moderateintensity aerobic exercise training regularly.

\section{Acknowledgments}

The authors like to thank Mekelle University for financial support.

\section{Declaration of Interest}

There is no conflict of interest.

\section{References}

Andreeva, O. (2014). Social and psychological factors that determine the recreational and health-improving activity of people of different age. Theory and Methods of Physical Education and Sport, 3, 35-40.

Baster, T., \& Baster-Brooks, C. (2005). Exercise and hypertension. Aust Fam Physician, 34 (6), 419-24.
Bateman, L.A., Slentz, C.A., Willis, L.H., Shields, T., Piner, L.W., Bales, C.W., Houmard, J.A., \& Kraus, W.E. (2011). Comparison of aerobic versus resistance exercise training effects on metabolic syndrome (from the studies of a targeted risk reduction intervention through defined exercise -STRRIDE-AT/RT). Am J Cardiol, 108, 838-44.

Belay, M.A., Reddy, R.C., \& Syam, B.M. (2013). The effects of combined aerobic and resistance exercise training on obese adults. Northwest Ethiopia. Research Journal of Recent Sciences, 2 (1), 59-66.

Boreham, C., \& Riddoch, C. (2001) The physical activity, fitness and health of children. J SportsSci, 19 (12), 91529.

Borjesson, M., Onerup, A., Lundqvist, S., \& Dahlof, B. (2016). Physical activity and exercise lower blood pressure in individuals with hypertension: narrative review of 27 RCTs. Br J Sports Med, 50 (6), 356-61.

Cardoso, C.D.Jr., Gomides, R.S., Queiroz, A.C., Pinto, L.G., da Silveira Lobo, F., Tinucci, T., Mion, D.Jr., \& de Moraes Forjaz, C.L. (2010). Acute and chronic effects of aerobic and resistance exercise on ambulatory blood pressure. Clinics (Sao Paulo), 65 (3), 317-25.

Carnethon, M.R., Gidding, S.S., Nehgme, R., Sidney, S., Jacobs, D.R.Jr., \& Liu, K. (2003). Cardio respiratory fitness in young adulthood and the development of cardiovascular risk factors. JAMA, 290, 3092-100.

Chobanian, A.V., Bakris, G.L., Black, H.R., Cushman, W.C., Green, L.A., Izzo, J.L., Jones, D.W., Materson, B.J., Oparil, S., Wright, J.T., \& Roccella, E.J. (2003). National heart, lung, and blood institute joint national committee on prevention, detection, evaluation, and treatment of high blood pressure; National high blood pressure education program coordinating committee. The seventh report of the joint national committee on prevention, detection, evaluation, and treatment of high blood pressure: the JNC 7 report. JAMA, 289 (19), 2560-72.

Collier, S.R., Kanaley, J.A., Carhart, Jr., Frechette, V., Tobin, M.M., Hall, A.K., Luckenbaugh, A.N., \& Fernhall, B. (2008). Effect of 4 weeks of aerobic or resistance exercise training on arterial stiffness, blood flow and blood pressure in pre- and stage-1 hypertensives. Journal of Human Hypertension, 22, 67886. 
Cornelissen, V.A., \& Fagard, R.H. (2005). Effects of endurance training on blood pressure, blood pressure regulating mechanisms and cardiovascular risk factors. J Hypertens, 45, 667-75.

Cornelissen, V.A., \& Smart, N.A. (2013). Exercise training for blood pressure: a systematic review and metaanalysis. J Am Heart Assoc, 2 (1), e004473.

Fagard, R.H. (2006). Exercise is good for your blood pressure: Effects of endurance training and resistance training. Clin Exp Pharmacol Physiol, 33 (9), 853-6.

Fagard, R.H., \& Cornelissen, V.A. (2007). Effect of exercise on blood pressure control in hypertensive patients. Eur J Cardiovasc Prev Rehabil, 14, 12-7.

Ferrari, C.K.B. (2008). Metabolic syndrome and obesity: Epidemiology and Prevention by physical activity and exercise. J Exerc Sci Fit, 6 (2), 87-96.

Gholamrezai, S., Zali, M., Shabani, R., \& Hoseini, R. (2014). Effect of 8 weeks of aerobic on body composition and blood pressure in postmenopausal women. Physical Education of Students, 5, 74-78.

Hailemariam, D., Kitaw, Y., Kaba, M., Siraw, D., Tebekaw, Y., Alemu, H. (2018). Ethiopia's urban primary health care reform: Practices, lessons, and the way forward. Ethiop J Health Dev, 32 (1), 4-9.

Halliwill, J.R., Buck, T.M., Lacewell, A.N., \& Romero, S.A. (2013). Post exercise hypotension and sustained post exercise vasodilatation: What happens after exercise? Exp Physiol, 98 (1), 7-18.

Hamer, M. (2006). The anti-hypertensive effects of exercise: Integrating acute and chronic mechanisms. Sports Med, 36 (2), 109-16.

Iellamo, F., \& Volterrani, M. (2010). Effect of exercise training in essential arterial hypertension. Rev Bras Hipertens, 17 (2), 68-71.

Izdebska, E., Cybulska, I., Izdebskir, J., Makowiecka, M., \& Trzebski, A. (2004). Effects of moderate physical training on blood pressure variability and hemodynamic pattern in mildly hypertensive subjects. J Physiol Pharmacol, 55 (4), 713-24.

Kearney, P.M., Whelton, M., Reynolds, K., Muntner, P., Whelton, P.K., \& He, J. (2005). Global burden of hypertension: analysis of worldwide data. Lancet, 365, 217-23.
Lamina, S. (2010). Effects of continuous and interval training programs in the management of hypertension: A randomized controlled trial. J Clin Hypertens (Greenwich), 12, 841-49.

Lima, A.H., Couto, H., Cardoso, G.A., Toscano, L.T., Silva, A.S., \& Mota, M.P. (2012). Aerobic training does not alter blood pressure in menopausal women with metabolic syndrome. Arq Bras Cardiol, 60 (3), 6-10.

Lima, L.G., Maria, J., Bonardi, T., Campos, G.O., Bertani, R.F., Scher, L.M., Moriguti, J.C., Ferriolli, E., \& Lima, N.K.C. (2014). Effect of an exercise program on blood pressure, body mass index and abdominal circumference in elderly hypertensive patients. J ASH, 8 (4S), e115-e118.

Maeda, S., Miyauchi, T., Kakiyama, T., Sugawara, J., Lemitsu, M., Irukayama-Tombe, Y., Murkami, H., Kumagai, Y., Kuno, S., \& Matsuda, M. (2001). Effects of exercise training of 8 weeks and detraining on plasma levels of endothelium-derived factors, endothelin-1 and nitric oxide, in healthy young humans. Life Sci, 69 (9), 1005-16.

Mills, K.T., Bundy, J.D., Kelly, T.N., Reed, J.E., Kearney, P.M., \& Reynolds, K. (2016). Global disparities of hypertension prevalence and control: A systematic analysis of population-based studies from 90 countries. Circulation, 134, 441-50.

Nshisso, L.D., Reesea, A., Gelayea, B., Lemma, S., Berhane, Y., \& Williams, M.A. (2012). Prevalence of hypertension and diabetes among Ethiopian adults. Diabetes Metab Syndr, 6 (1), 36-41.

O'Sullivan SE, Bell C. The effects of exercise and training on human cardiovascular reflex control. J Auton Nerv Syst, 2000; 81(3): 16-24.

Parkinson, C.F. (2012). Study guide for understanding pathophysiology. 5th ed., Missouri: Elsevier, 2012

Pescatello, L.S., MacDonald, H.V., Lambeti, L., \& Johnson, B.T. (2015). Exercise for hypertension: a prescription update integrating existing recommendations with emerging research. Curr Hypertens Rep, 17 (87), 1-10.

Swati, S., Pawaria, S., \& Sheetal, K. (2015). Effects of aerobic versus resistance training on blood pressure in hypertensive patients. J Anesth Crit Care Open Access, 3 (3), 1-6.

Tsai, J., Liu, J.C., Kao, C.C., Tomlinson, B., Kao, P.F., Chen, J.W., \& Chan, P. (2002). Beneficial effects on blood 
pressure and lipid profile of programmed exercise training in subjects with white coat hypertension. Am J Hypertens, 15, 571-76.

Tsai, J.C., Yang, H.Y., Wang, W.H., Hsieh, M.H., Chen, P.T., Kao, C.C., Kao, P.F., Wang, C.H., \& Chan, P. (2004). The Beneficial effect of regular endurance exercise training on blood pressure and quality of life in patients with hypertension. Clinical and Experimental Hypertension, 26 (3), 255-65.

Westhoff, T.H., Schmidt, S., Gross, V., Joppke, M., Zidek, W., der Giet, M.V., \& Dimeob, F. (2008). The cardiovascular effects of upper-limb aerobic exercise in hypertensive patients. Journal of Hypertension, 26 (7), 1336-42.
Whelton, P.K., He, J., Appel, L.J., Cutler, J.A., Havas, S., Kotchen, T.A., Roccella, E.J., Stout, R., Vallbona, C., Winston, M.C., \& Karimbakas, J. (2002a). National High Blood Pressure Education Program Coordinating Committee. Primary prevention of hypertension: Clinical and public health advisory from the national high blood pressure education program. JAMA, 288, 1882-8.

Whelton, S.P., Chin, A., Xin, X., \& He, J. (2002b). Effect of Aerobic Exercise on Blood Pressure: A Meta-Analysis of Randomized, Controlled Trials. Ann Intern Med, 136 (7), 493-503.

WHO. (2011). Global Status Report on Non communicable Diseases 2010. Geneva: World Health Organization, 2011. http://www.who.int /nmh/publications/ncd_ report2010/en (accessed May 16, 2017). 